\title{
Comparison of Native Escherichia coli L-Asparaginase versus Pegylated Asparaginase, in Combination with Ifosfamide, Methotrexate, Etoposide, and Prednisolone, in Extranodal NK/T-Cell Lymphoma, Nasal Type
}

\author{
Hyun Jee Kim, MS ${ }^{\uparrow}$ \\ Chan-Young Ock, MD2 \\ Tae Min Kim, MD, PhD2,3 \\ Sung Hee Lee, BS ${ }^{1}$ \\ Ju-Yeun Lee, $\mathrm{PhD}^{4}$ \\ Sun hoi Jung, $P h D^{1}$ \\ Yoon Sook Cho, PhD \\ Miso Kim, MD2,3 \\ Bhumsuk Keam, MD, PhD 2,3 \\ Dong-Wan Kim, MD, PhD2,3 \\ II Han Kim, MD, PhD 5 \\ Dae Seog Heo, MD, PhD'2,3
}

Departments of ${ }^{1}$ Pharmacy and ${ }^{2}$ Internal Medicine, Seoul National University Hospital, Seoul, ${ }^{3}$ Seoul National University Cancer Research Institute, Seoul, ${ }^{4}$ College of Pharmacy and Institute of Pharmaceutical Science and Technology, Hanyang University, Guri, ${ }^{5}$ Department of Radiation Oncology, Seoul National University Hospital, Seoul, Korea

\section{Purpose}

The aim of this study was to compare asparaginase-related toxicities in two asparaginase preparations, namely native Escherichia coli L-asparaginase (L-ASP) and pegylated asparaginase (PEG-ASP) in combination with ifosfamide, methotrexate, etoposide, and prednisolone (IMEP) in natural killer (NK)/T-cell lymphoma (NTCL).

\section{Materials and Methods}

A total of 41 NTCL patients who received IMEP plus native E. coli L-ASP or PEG-ASP at Seoul National University Hospital were included in this study between January 2013 and March 2016. IMEP/ASP treatment consisted of ifosfamide, methotrexate, etoposide, plus native $E$. coli L-ASP $\left(6,000 \mathrm{IU} / \mathrm{m}^{2}\right.$ on days $1,3,5,7,9$, and 11$)$ or PEG-ASP $\left(2,500 \mathrm{IU} / \mathrm{m}^{2}\right.$ on day 1$)$ every 3 weeks. ASP-related toxicities, toxicity patterns, length of hospital stay, and clinical outcomes were compared between the different treatment groups.

\section{Results}

The frequency of ASP-related toxicities was similar between the IMEP plus native $E$. coli L-ASP group and the PEG-ASP group apart from hypofibrinogenemia (native E. coli L-ASP vs. PEG-ASP group, $86.4 \%$ vs. $36.8 \%$; $p=0.001$ ). Although post-treatment transaminase and albumin levels were significantly high and low, respectively, hepatotoxicity gradients before and after treatment did not differ significantly between the groups. Since PEG-ASP was given at an outpatient clinic in some patients, length of hospital stay was significantly shorter in the IMEP plus PEG-ASP group (median, 4.0 vs. 6.0 days; $p=0.002$ ). A favorable tendency of clinical outcomes was observed in NTCL patients treated with IMEP plus PEG-ASP (complete remission rate, $73.7 \%$ vs. $45.5 \%$; $p=0.067$ ).

\section{Conclusion}

IMEP plus PEG-ASP showed similar ASP-related toxicities, shorter length of hospital stay, and a trend towards improved clinical outcomes compared with IMEP plus native $E$. coli L-ASP in NTCL.
Correspondence: Tae Min Kim, MD, PhD

Department of Internal Medicine,

Seoul National University Hospital,

101 Daehak-ro, Jongno-gu, Seoul 03080, Korea

Tel: 82-2-2072-3559

Fax: 82-2-762-9662

E-mail: gabrie19@snu.ac.kr

Received January 26, 2017

Accepted June 26, 2017

Published Online July 3, 2017

*Hyun Jee Kim and Chan-Young Ock

contributed equally to this work.
Key words

NK/T-cell lymphoma, Native Escherichia coli L-asparaginase,

Pegylated asparaginase, Toxicity, Length of stay 


\section{Introduction}

Natural killer (NK) / T-cell lymphoma (NTCL) is a distinct clinicopathologic disease, as defined by the World Health Organization (WHO), displaying very aggressive features of lymphoproliferative diseases [1]. Although long-term outcomes with conventional combination chemotherapy have been unsatisfactory in the past [2,3], recent phase II studies have demonstrated the efficacy of L-asparaginase-based combination chemotherapy with a backbone of ifosfamide, methotrexate, etoposide, and prednisolone (IMEP) in newly diagnosed stage IV or refractory NTCL [4-6].

In Korea, three preparations of asparaginase for treating NTCL are available: native Escherichia coli L-asparaginase (L-ASP), namely pegylated E. coli asparaginase (PEG-ASP), and Erwinia-derived asparaginase. Generally, the E. coliderived enzyme is used as front-line therapy, and the Erwinia-derived preparation is only reserved for patients who develop hypersensitivity reactions $[7,8]$. However, native $E$. coli L-ASP is associated with notable side-effects including hypersensitivity, pancreatitis, and thrombosis [7-12]. Hypersensitivity to native E. coli L-ASP has resulted in a poorer outcome due to inactivation of asparaginase by antibodies [13]. PEG-ASP is potentially less immunogenic than native E. coli L-ASP and has a longer half-life, which allows one injection of PEG-ASP to replace multiple injections of native E. coli L-ASP [14,15]. PEG-ASP is now being used as the initial asparaginase preparation in some pediatric acute lymphoblastic leukemia (ALL) treatment regimens after randomized trials have confirmed that it has similar toxicity and efficacy to native E. coli L-ASP $[16,17]$.

Although PEG-ASP has been used as the initial asparaginase preparation in some prospective NTCL clinical trials in China $[18,19]$ and the pharmacokinetics of each asparaginase preparation have been well-defined in many reports [20-22], there are no studies that compare the ASP-related toxicity of PEG-ASP with native E. coli L-ASP in the NTCL population. In addition, the length of hospital stay and number of visits to the emergency room (ER) after chemotherapy with each drug preparation have not been clearly reported. The aim of this study was to compare ASP-related toxicity between two asparaginase preparations, namely native E. coli L-ASP and PEG-ASP, in combination with IMEP in NTCL.

\section{Materials and Methods}

\section{Patients}

A total of 41 patients who were treated with IMEP plus native E. coli L-ASP or PEG-ASP for NTCL between January 2013 and March 2016 at Seoul National University Hospital were retrospectively identified. The inclusion criteria were AS follows: (1) pathologically confirmed NTCL according to the WHO criteria [1,23]; (2) treatment with IMEP plus native E. coli L-ASP or PEG-ASP; and (3) eligibility for ASP-related toxicity evaluation after chemotherapy. Clinical demographics including age, sex, B symptoms, Eastern Cooperative Oncology Group performance status (ECOG PS), serum lactate dehydrogenase level, Ann Arbor stage, number of extranodal sites, International Prognostic Index (IPI) score, Korean Prognostic Index (KPI) score, Prognostic Index of Natural Killer Lymphoma (PINK) score, Prognostic Index of Natural Killer Lymphoma-Epstein-Barr virus (PINK/E) score, and previous lines of chemotherapy, were recorded [24,25].

\section{Treatment}

All patients received chemotherapy consisting of IMEP, plus native E. coli L-ASP or PEG-ASP. The choice of asparaginase preparation in combination with the IMEP regimen was made by the primary physician. The conventional IMEP regimen consists of ifosfamide at a dose of $1.5 \mathrm{~g} / \mathrm{m}^{2}$, administered intravenously on days 1 to 3 with adequate hydration of $2 \mathrm{~L}$ of half-saline per day and mesna to prevent hemorrhagic cystitis; methotrexate at a dose of $30 \mathrm{mg} / \mathrm{m}^{2}$, given intravenously on day 4; and etoposide at a dose of 100 $\mathrm{mg} / \mathrm{m}^{2}$, administered intravenously on days 1 to 3 . In the IMEP plus L-ASP group, prednisolone was given at a dose of $50 \mathrm{mg} / \mathrm{m}^{2}$ orally on days 1 to 5 . In the IMEP plus PEGASP group, dexamethasone was given at a dose of $20 \mathrm{mg}$ intravenously on days 1 to 4 . Patients received either intravenous native $E$. coli L-ASP 6,000 IU / m² (capped at 10,000 IU) on days $1,3,5,7,9$, and 11 or intravenous PEG-ASP 2,500 $\mathrm{IU} / \mathrm{m}^{2}$ (capped at 3,750 IU) on day 1 of each cycle. Each cycle of chemotherapy was given every 3 weeks. For patients with stage I/II NTCL, up to three-to-four cycles of treatment were planned, followed by involved-field radiotherapy (IFRT). For patients with stage III/IV NTCL, up to six cycles of treatment were planned. Treatment was continued until disease progression, unacceptable toxicities, or refusal by the patient. To minimize allergic reactions with native E. coli L-ASP, all patients were allergy tested at 1 hour prior to receiving native E. coli L-ASP at our institution. In cases of positive skin test and hypersensitivity to L-ASP, patients were required to receive native $E$. coli L-ASP under a desensitization protocol. 


\section{Asparaginase-related toxicity assessment}

Toxicity was assessed according to the Common Terminology Criteria for Adverse Events, ver. 3.0. Asparaginaserelated toxicities were defined as toxicities that are caused by asparagine depletion and have been commonly reported in clinical trials in combination with IMEP in NTCL [4-6]; these included hepatotoxicity (measured by elevated levels of aspartate aminotransferase (AST), alanine aminotransferase (ALT), alkaline phosphatase (ALP), and total bilirubin (t-bil), hypoalbuminemia, hypofibrinogenemia; all grades), hyperglycemia, hypertriglycemia (all grades), pancreatitis (grade 2 or worse), and allergy (all grades, including anaphylaxis). For patients with no history of diabetes mellitus, hyperglycemia was defined as two or more random blood glucose levels higher than $200 \mathrm{mg} / \mathrm{dL}$. For patients with underlying diabetes mellitus, prescription of new hyperglycemia medication or increase in the dose of hyperglycemia medication after starting treatment with IMEP plus native E. coli L-ASP or PEG-ASP was considered as an event of hyperglycemia. Hematological toxicities were not included because these toxicities are routinely encountered at diagnosis and during treatment of NTCL. Toxicity was continually monitored for one cycle up to the subsequent cycle of chemotherapy, initiation of a different chemotherapy regimen due to disease progression, or death.

To compare toxicity patterns between the two groups after the first cycle and during chemotherapy, we observed changes in AST, ALT, ALP, t-bil, and albumin between day 1 of the first cycle and 3 weeks after the last cycle of chemotherapy. We calculated absolute change and percentage change in laboratory values ( $\triangle \mathrm{AST}, \triangle \mathrm{ALT}, \triangle \mathrm{ALP}, \Delta \mathrm{t}$-bil, and $\Delta$ albumin).

\section{Length of hospital stay and clinical outcomes}

The length of hospital stay and number of visits to the ER were compared after each cycle of IMEP plus native E. coli L-ASP or PEG-ASP. Clinical outcomes were assessed by physical examination, otolaryngologic examination, computed tomography scans, and positron emission tomography (PET) scans, using the response criteria for lymphoma [26].

Briefly, complete response (CR) was defined as disappearance of all evidence of disease with no PET-positive lesions. Partial response (PR) was defined as at least 50\% decrease in the sum of the product of the diameters of up to six dominant masses, and no increase in size of other nodes. Progressive disease (PD) was defined as any new lesion or at least $50 \%$ increase of any single previously identified node and stable disease is defined as failure to attain the criteria needed for a CR or PR, but doe s not fulfill those of PD. Responses were assessed every two cycles during chemotherapy and best responses to chemotherapy were used in the analysis. Overall survival (OS) and progression-free survival (PFS) were calculated as the time from the date of the first administration of IMEP plus native E. coli L-ASP or PEG-ASP up to the date of either death or the final follow-up visit, and to the date of disease progression, respectively, confirmed by the response assessment of lymphoma [26].

\section{Statistical analysis}

Clinical variables were compared between the two groups using the Pearson chi-square or Fisher exact tests for categorical variables, and by the Wilcoxon rank-sum test for continuous variables. We used multivariable logistic regression to adjust for baseline differences when comparing asparaginase-related toxicity, where necessary. Percentage changes in laboratory values ( $\triangle \mathrm{AST}, \triangle \mathrm{ALT}, \triangle \mathrm{ALP}, \Delta \mathrm{t}$-bil, $\Delta$ albumin, and $\Delta$ fibrinogen) before and after chemotherapy were compared between the two groups using the Wilcoxon signed rank test. Survival curves were derived by the Kaplan-Meier method and compared using a log-rank test. A significance level of $<0.05$ was used for $p$-values. All analyses were performed using SPSS ver. 22.0 (IBM Corp., Armonk, NY) and STATA ver. 12 (StataCorp LP, College Station, TX).

\section{Ethical statement}

This study was approved by the Institutional Review Board of Seoul National University Hospital (H-1508-177702), and was conducted in accordance with the precepts established by the Declaration of Helsinki.

\section{Results}

\section{Patients' characteristics and treatments}

A total of 22 patients received IMEP plus native E. coli L-ASP for 77 cycles, and 19 patients received IMEP plus PEGASP for 54 cycles. All clinical demographics were similar between the L-ASP and PEG-ASP groups. The median age was 58.8 years in the L-ASP group and 55.9 years in the PEGASP group. A total of $11(50.0 \%)$ patients in the L-ASP group and nine $(47.4 \%)$ patients in the PEG-ASP group had Ann Arbor stage III/IV NTCL. Patients in each group were well balanced according to the PINK and PINK/E model. A total of $15(68.2 \%)$ and 14 (73.7\%) NTCL patients received IMEP plus L-ASP or IMEP plus PEG-ASP, respectively, as a firstline therapy. Baseline laboratory values were similar between the two groups (Table 1). 
Table 1. Baseline patient characteristics

\begin{tabular}{|c|c|c|c|}
\hline & IMEP+L (n=22) & $\mathrm{IMEP}+\mathrm{P}(\mathrm{n}=19)$ & p-value \\
\hline \multicolumn{4}{|l|}{ Age (yr) } \\
\hline$<60$ & $12(54.5)$ & $13(68.4)$ & 0.364 \\
\hline$\geq 60$ & $10(45.5)$ & $6(31.6)$ & \\
\hline Median $(75 \% \mathrm{CI})$ & $58.8(44.4-68.8)$ & $55.9(49.8-63.6)$ & 0.896 \\
\hline \multicolumn{4}{|l|}{ Sex } \\
\hline Male & $12(54.5)$ & $8(42.1)$ & 0.427 \\
\hline Female & $10(45.5)$ & $11(57.9)$ & \\
\hline \multicolumn{4}{|l|}{ Presentation } \\
\hline UAT & $19(86.4)$ & $14(73.7)$ & 0.436 \\
\hline Not UAT & $3(13.6)$ & $5(26.3)$ & \\
\hline \multicolumn{4}{|l|}{ B symptoms } \\
\hline No & $15(68.2)$ & $17(89.5)$ & 0.140 \\
\hline Yes & $7(31.8)$ & $2(10.5)$ & \\
\hline \multicolumn{4}{|l|}{ Ann Arbor stage } \\
\hline I-II & $11(50.0)$ & $10(52.6)$ & 0.867 \\
\hline III-IV & $11(50.0)$ & $9(47.4)$ & \\
\hline \multicolumn{4}{|l|}{ LDH level } \\
\hline Normal & $11(50.0)$ & $13(68.4)$ & 0.233 \\
\hline Elevated & $11(50.0)$ & $6(31.6)$ & \\
\hline \multicolumn{4}{|l|}{ No. of extranodal sites } \\
\hline $0-1$ & $17(77.3)$ & $16(84.2)$ & 0.703 \\
\hline$\geq 2$ & $5(22.7)$ & $3(15.8)$ & \\
\hline \multicolumn{4}{|l|}{ ECOG PS } \\
\hline $0-1$ & $20(90.9)$ & $16(84.2)$ & 0.649 \\
\hline$\geq 2$ & $2(9.1)$ & $3(15.8)$ & \\
\hline \multicolumn{4}{|l|}{ IPI score } \\
\hline $0-1$ & $11(50.0)$ & $10(52.6)$ & 0.300 \\
\hline 2 & $4(18.2)$ & $7(36.8)$ & \\
\hline 3 & $5(22.7)$ & $1(5.3)$ & \\
\hline $4-5$ & $2(9.1)$ & $1(5.3)$ & \\
\hline \multicolumn{4}{|l|}{ KPI score } \\
\hline 1 & 4 & 2 & 0.006 \\
\hline 2 & 7 & 6 & \\
\hline 3 & 1 & 9 & \\
\hline 4 & 10 & 2 & \\
\hline \multicolumn{4}{|l|}{ PINK } \\
\hline Low & 4 & 4 & 0.917 \\
\hline Intermediate & 13 & 10 & \\
\hline High & 5 & 5 & \\
\hline \multicolumn{4}{|l|}{ PINK/E } \\
\hline Low & 5 & 4 & 0.977 \\
\hline Intermediate & 12 & 11 & \\
\hline High & 5 & 4 & \\
\hline \multicolumn{4}{|l|}{ Previous chemotherapy } \\
\hline 0 & $15(68.2)$ & $14(73.7)$ & 0.634 \\
\hline $1-2$ & $6(27.3)$ & $5(26.3)$ & \\
\hline 3 & $1(4.5)$ & 0 & \\
\hline AST, median $(75 \% \mathrm{CI})$ & $33.50(21.75-110.25)$ & $22.0(17.0-49.0)$ & 0.169 \\
\hline ALT, median $(75 \% \mathrm{CI})$ & $41.50(19.50-62.75)$ & $20.0(13.0-47.0)$ & 0.089 \\
\hline ALP, median $(75 \% \mathrm{CI})$ & $62.50(52.25-132.25)$ & $94.0(79.0-133.0)$ & 0.075 \\
\hline t-bil, median $(75 \% \mathrm{CI})$ & $0.65(0.40-1.00)$ & $0.5(0.4-0.7)$ & 0.323 \\
\hline
\end{tabular}

(Continued to the next page) 
Table 1. Continued

\begin{tabular}{lccc} 
& IMEP+L (n=22) & IMEP+P (n=19) & p-value \\
\hline Albumin, median $(\mathbf{7 5} \% \mathbf{C I})$ & $3.65(3.175-4.15)$ & $3.8(3.1-4.2)$ & 0.733 \\
\hline Fibrinogen, median $(\mathbf{7 5} \% \mathbf{C I})^{\mathrm{a})}$ & $295.0(214.5-392)$ & $321.0(169.0-397.0)$ & 0.959 \\
\hline
\end{tabular}

$\mathrm{IMEP}+\mathrm{L} / \mathrm{P}$, ifosfamide, mesna, etoposide, prednisolone+L-asparaginase/peg-asparaginase; CI, confidence interval; UAT, upper aerodigestive tract; LDH, lactate dehydrogenase; ECOG PS, Eastern Cooperative Oncology Group performance status; IPI, International Prognostic Index; KPI, Korean Prognostic Index; PINK, Prognostic Index of Natural Killer Lymphoma; PINK/E, PINK-Epstein-Barr virus; AST, aspartate aminotransferase; ALT, alanine aminotransferase; ALP, alkaline phosphatase; t-bil, total bilirubin. ${ }^{a}$ Evaluated in 27 patients.

Table 2. Asparaginase-related toxicity per patient

\begin{tabular}{|c|c|c|c|}
\hline & IMEP+L (n=22) & IMEP+P (n=19) & p-value \\
\hline \multicolumn{4}{|c|}{ Asparaginase-related toxicity (all grades) } \\
\hline AST elevation & $10(45.5)$ & $9(47.4)$ & 0.902 \\
\hline ALT elevation & $11(50.0)$ & $12(63.2)$ & 0.397 \\
\hline ALP elevation & $8(36.4)$ & $11(57.9)$ & 0.168 \\
\hline t-bil elevation & $13(59.1)$ & $8(42.1)$ & 0.278 \\
\hline Hypoalbuminemia & $16(72.7)$ & $13(68.4)$ & 0.763 \\
\hline Hypofibrinogenemia & $19(86.4)$ & $7(36.8)$ & 0.001 \\
\hline Hyperglycemia & $5(22.7)$ & $1(5.3)$ & 0.112 \\
\hline Hypertriglycemia & $6(27.3)$ & $4(21.1)$ & 0.727 \\
\hline Pancreatitis & $1(4.5)$ & $2(10.5)$ & 0.588 \\
\hline Allergy & $7(31.8)$ & $3(15.8)$ & 0.366 \\
\hline \multicolumn{4}{|c|}{ Asparaginase-related toxicity ( $\geq$ grades 3 ) } \\
\hline AST elevation & $3(13.6)$ & $2(10.5)$ & $>0.99$ \\
\hline ALT elevation & $1(4.5)$ & $2(10.5)$ & 0.588 \\
\hline ALP elevation & $1(4.5)$ & 0 & $>0.99$ \\
\hline t-bil elevation & $2(9.1)$ & 0 & 0.490 \\
\hline Hypoalbuminemia & $1(4.5)$ & $1(5.3)$ & $>0.99$ \\
\hline Hypofibrinogenemia & $3(13.6)$ & $3(15.8)$ & $>0.99$ \\
\hline Allergy & $2(9.1)$ & $1(5.3)$ & 0.485 \\
\hline
\end{tabular}

$\mathrm{IMEP}+\mathrm{L} / \mathrm{P}$, ifosfamide, mesna, etoposide, prednisolone+L-asparaginase/peg-asparaginase; AST, aspartate aminotransferase; ALT, alanine aminotransferase; ALP, alkaline phosphatase; t-bil, total bilirubin.

In L-ASP group, seven patients with stage I/II and five patients with stage III/ IV completed the planned chemotherapy. Ten patients discontinued chemotherapy due to death $(n=2)$, disease progression $(n=5)$, and unacceptable toxicities $(n=3)$. In PEG-ASP group, seven patients with stage I/ II and five patients with stage III/IV completed the planned chemotherapy. Seven patients discontinued chemotherapy due to death $(n=1)$, unacceptable toxicities $(n=5)$, and refusal by the patient $(n=1)$. Eight patients in L-ASP group and seven patients in PEG-ASP group received sequential IFRT at median doses of $40.5 \mathrm{~Gy}$ and $42.0 \mathrm{~Gy}$, respectively. Three patients in L-ASP group and two patients in PEG-ASP group received autologous stem-cell transplantation and allogenic stem-cell transplantation as consolidation, respectively.

\section{Asparaginase-related toxicity assessment}

The asparaginase-related toxicity of the current study is summarized in Table 2. Hepatotoxicity was the most frequent asparaginase-related toxicity in both the L-ASP and PEG-ASP groups. There were no significant differences between the levels of AST, ALT, ALP and t-bil, or hypoalbuminemia between the two groups. However, more patients in the L-ASP group experienced hypofibrinogenemia com- 


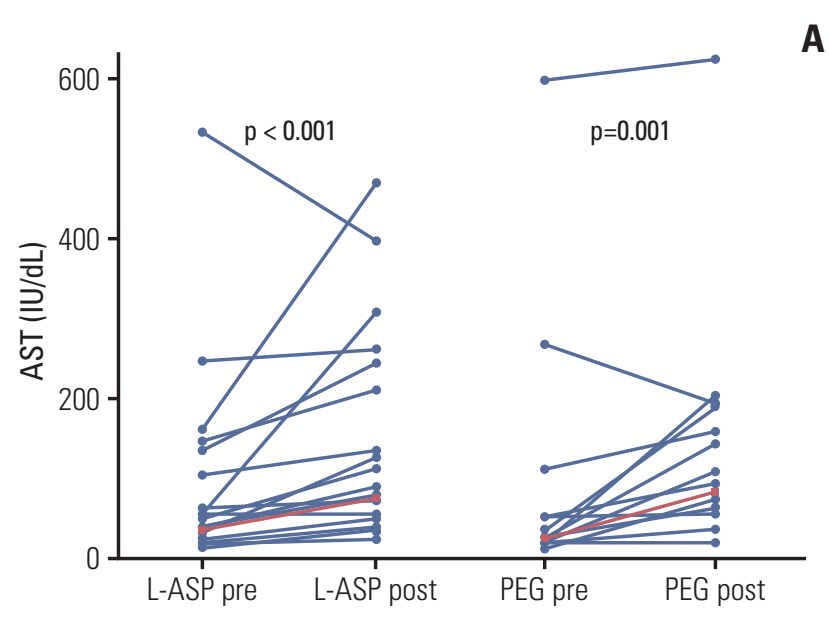

A
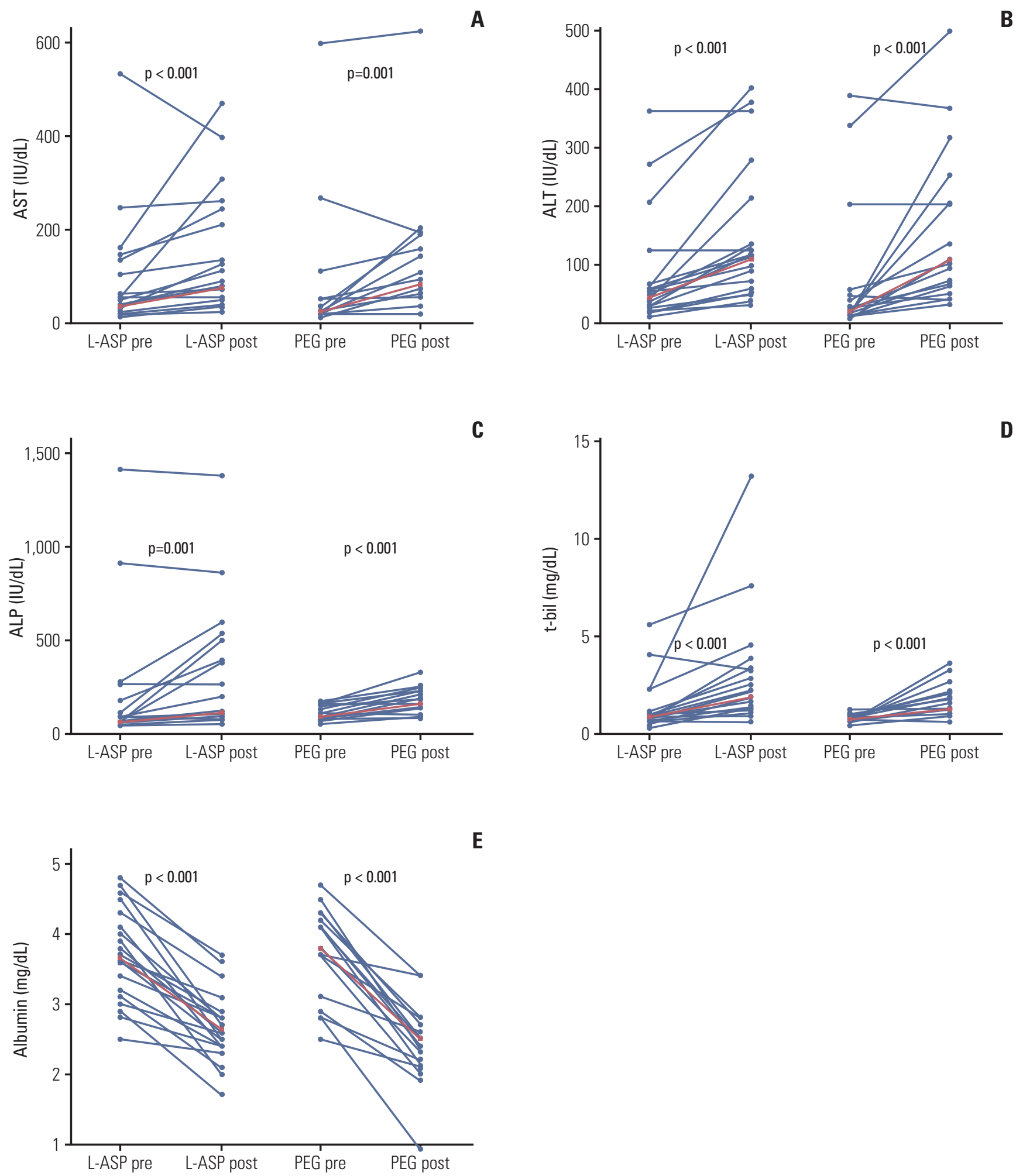

Fig. 1. Comparison of liver function tests between before chemotherapy (pre) and maximum values after chemotherapy (post). Each dot represents pre and post aspartate aminotransferase (AST) (A), alanine aminotransferase (ALT) (B), alkaline phosphatase (ALP) (C), total bilirubin (t-bil) (D), and albumin (E) levels. The red line represents the change in median pre and post values. Pre and post values were compared within each group using the Wilcoxon signed rank test. L-ASP, L-asparaginase; PEG, pegylated. 

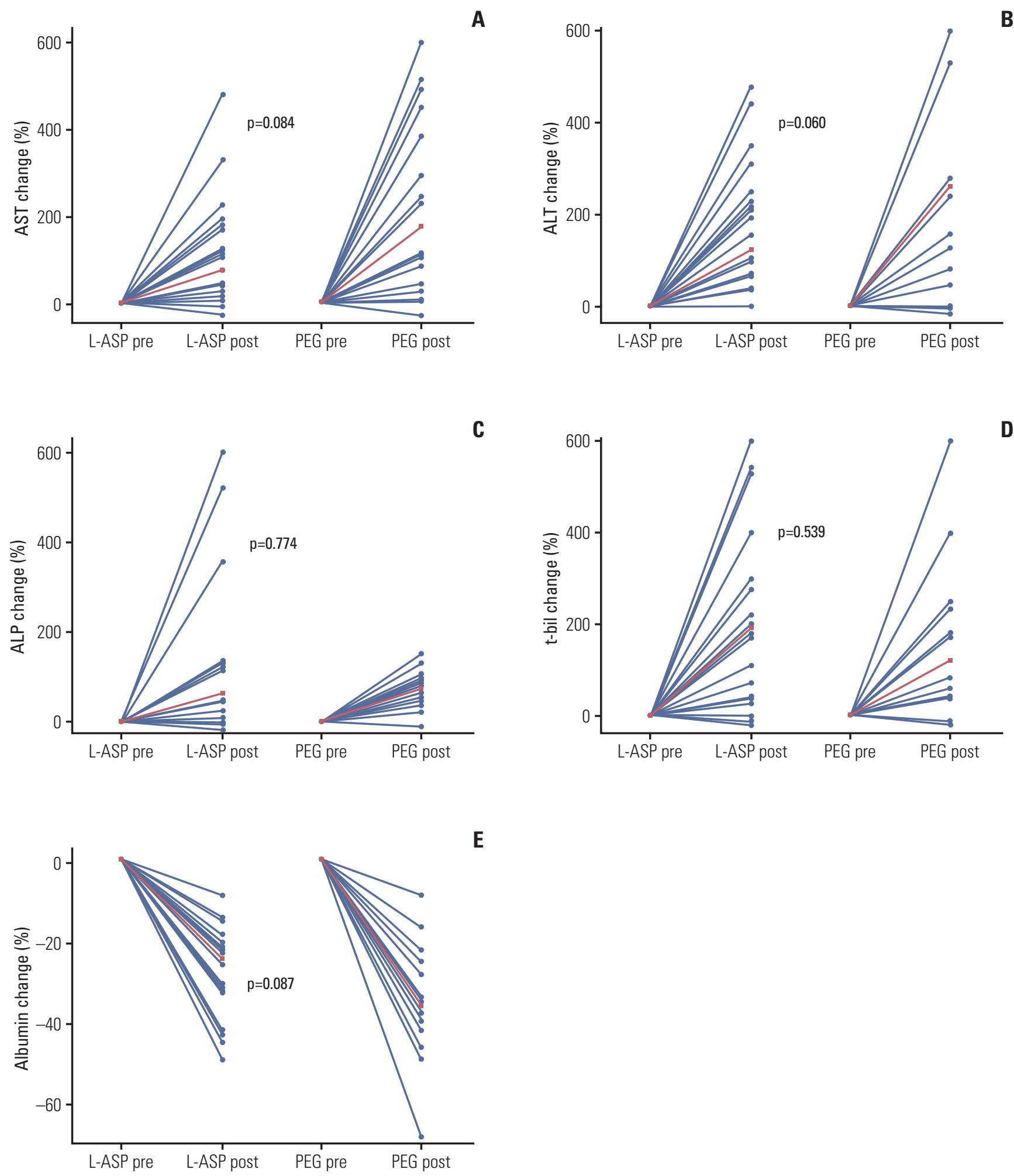

Fig. 2. Comparison of percentage changes in liver function tests between before chemotherapy (pre) and maximum values after (post) chemotherapy. Each dot represents the percentage change between pre and post aspartate aminotransferase (AST) (A), alanine aminotransferase (ALT) (B), alkaline phosphatase (ALP) (C), total bilirubin (t-bil) (D), and albumin (E) levels. The red line represents the change in median pre and post percentage change. Percentage change between the two groups was compared by the Wilcoxon signed rank test. L-ASP, L-asparaginase; PEG, pegylated. 
Table 3. Length of hospital stay and number of ER visits

\begin{tabular}{lccc} 
& IMEP+L (77 cycles) & IMEP+P (54 cycles) & p-value \\
Hospital stay, median $(75 \%$ CI) & $6.0(4.0-13.0)$ & $4.0(2.25-7.25)$ & 0.002 \\
ER visit, $\mathrm{n}(\%)$ & $3(3.9)$ & $6(11.1)$ & 0.160 \\
\hline
\end{tabular}

$\mathrm{ER}$, emergency room; IMEP+L/P, ifosfamide, mesna, etoposide, prednisolone+L-asparaginase/peg-asparaginase; $\mathrm{CI}$, confidence interval.

Table 4. Clinical outcomes of IMEP plus native Escherichia coli L-ASP and PEG-ASP

\begin{tabular}{lccc} 
Response assessment & IMEP+L (n=21) & IMEP+P (n=18) & p-value \\
PR & $5(23.8)$ & $4(22.2)$ & $>0.99$ \\
CR & $10(47.6)$ & $14(77.8)$ & 0.098 \\
PD & $5(23.8)$ & 0 & 0.050 \\
SD & $1(4.8)$ & 0 & $>0.99$ \\
\hline
\end{tabular}

Values are presented as number (\%). IMEP, ifosfamide, mesna, etoposide, prednisolone; L-ASP, L-asparaginase; PEG-ASP, pegylated asparaginase; IMEP $+\mathrm{L} / \mathrm{P}$, ifosfamide, mesna, etoposide, prednisolone $+\mathrm{L}$-asparaginase/peg-asparaginase; $\mathrm{PR}$, partial remission; $\mathrm{CR}$, complete remission; $\mathrm{PD}$, progressive disease; $\mathrm{SD}$, stable disease.

pared to the PEG-ASP group ( $86.4 \%$ vs. $36.8 \%$, $\mathrm{p}=0.001)$.

More patients in the L-ASP group experienced an allergic reaction, although the difference was not significant. In our study, three patients (13.6\%) taking L-ASP had a positive skin test prior to receiving native E. coli L-ASP. All three patients received native $E$. coli L-ASP under a desensitization protocol, and two patients completed all subsequent cycles of native E. coli L-ASP under the desensitization protocol without allergic toxicities. However, one patient experienced a grade 3 allergic reaction despite the desensitization protocol and, therefore, switched to Erwinia-derived asparaginase. A grade 3 allergic reaction occurred in one patient on L-ASP despite a negative skin test result, and this patient completed all subsequent cycles of IMEP plus L-ASP under a desensitization protocol. One patient treated with IMEP plus PEGASP experienced an asparaginase-related grade 3 allergic reaction and, as a result, discontinued this drug from subsequent cycles of chemotherapy.

There was no significant difference in the frequency of grade 3 or above asparaginase-related toxicity between the two groups. Two patients experienced grade 4 asparaginaserelated toxicity; one patient developed grade 4 hypertriglyceridemia after six cycles of IMEP plus L-ASP, and another patient experienced grade 4 hypofibrinogenemia after two cycles of IMEP plus PEG-ASP. These latter two adverse events were not life-threatening and soon resolved after chemotherapy was completed. There were no treatmentrelated deaths in this study.

Upon analysis of the asparaginase-related toxicity per cycle of chemotherapy, the frequency of toxicity in each group was found to be comparable. However, hyperglycemia and allergy occurred more frequently in the L-ASP group ( $\mathrm{p}=0.045$ and $\mathrm{p}=0.005$, respectively). Frequency of grade 3 or above asparaginase-related toxicity per cycle of chemotherapy did not show any significant difference between the two groups (S1 Table).

The level of changes in hepatic enzymes, bilirubin, and albumin between native E. coli L-ASP and PEG-ASP after chemotherapy were similar in two groups. Patients who received IMEP plus L-ASP and PEG-ASP displayed significantly elevated levels of AST, ALT, ALP, and t-bil and decreased level of albumin after chemotherapy compared to before chemotherapy $(p<0.05)$ (Fig. 1). When we compared the percent change of AST, ALT, ALP, t-bil, and albumin before and after chemotherapy, level of changes were similar between two groups (Fig. 2). However the toxicity pattern after the first cycle of chemotherapy was significantly different. Only the patients who received IMEP plus PEG-ASP showed significantly elevated levels of AST, ALT, ALP, and t-bil after one cycle of chemotherapy compared to day 1 (AST, $\mathrm{p}=0.024 ;$ ALT, $\mathrm{p}=0.033$; ALP, $\mathrm{p}<0.001$; and t-bil, $\mathrm{p}<0.001)$. Although the level of albumin significantly decreased in both groups, the slope of change was more significant in the PEG-ASP group (in both groups $\mathrm{p}<0.001$ ) (S2 Fig.). When we compared the percentage change of AST, ALT, ALP, t-bil, and albumin between the two groups on day 1 of the first cycle and 3 weeks after the first cycle, patients in the PEG-ASP group displayed significantly elevated levels 
of AST, ALT, and t-bil compared to the L-ASP group (AST, $\mathrm{p}=0.036$; ALT, $\mathrm{p}=0.034$; and t-bil, $\mathrm{p}<0.001$ ) (S3 Fig.).

\section{Length of hospital stay and dose profile of chemother- apy}

We compared the length of hospital stay and number of visits to the ER between the L-ASP and PEG-ASP groups after each cycle of chemotherapy. The number of ER visits was not significantly different between the two groups. Five patients received 13 cycles of IMEP plus PEG-ASP at an outpatient clinic leading to significantly shorter hospital stays in PEG-ASP group compared to L-ASP group (median, 4.0 days vs. 6.0 days; $p=0.002$ ) (Table 3 ).

In order to investigate the effect of asparaginase-related toxicity on the dose profile of chemotherapy, we analyzed the number of cycles with reduced doses, the amount of dose reduction, and the number of completed injections of L-ASP or PEG-ASP according to the planned treatment course. The number of cycles with reduced doses and the amount of dose reduction did not show any significant difference between the two groups. Native E. coli L-ASP was given according to the planned treatment course in only 62 cycles $(80.5 \%)$, whereas PEG-ASP was given according to the planned treatment course in all cycles $(\mathrm{p}<0.001)$. Native E.coli L-ASP was incompletely injected in 15 cycles due to toxicities and intolerance (S4 Table).

\section{Clinical outcomes and survival analysis}

Thirty-nine patients were eligible for response evaluation. The PEG-ASP group showed a tendency towards better clinical outcome compared to the L-ASP group in NTCL. Five patients $(23.8 \%)$ in the L-ASP group and four patients $(22.2 \%)$ in the PEG-ASP group achieved a PR after chemotherapy. The number of patients who achieved a CR was different between the two groups, although the difference was not statistically significant: 10 patients $(47.6 \%)$ in the L-ASP group and 14 patients (77.8\%) in the PEG-ASP group. Although five patients (23.8\%) in L-ASP group showed disease progression, none in PEG-ASP group showed disease progression during the follow-up period (Table 4).

After a median follow up of 23.3 months (range, 1.3 to 46.7 months), median PFS and OS were not significantly different between the two groups regardless of the Ann Arbor stage in patients who received the first-line IMEP plus L-ASP or PEG-ASP (S5 Fig.). High IPI, KPI, and PINK/E scores reduced survivals in NTCL patients (S6 Fig.).

\section{Discussion}

Our results show that native E. coli L-ASP and PEG-ASP showed a similar frequency of asparaginase-related toxicity in combination with IMEP in NTCL. Hypofibrinogenemia was the only asparaginase-related toxicity that differed in frequency between the L-ASP and the PEG-ASP groups. However, the toxicity patterns were significantly different in the two groups. After the first cycle of IMEP plus PEG-ASP, patients displayed significantly elevated levels of AST, ALT, ALP, and t-bil whereas the level of albumin decreased significantly. Patients who received IMEP plus PEG-ASP seemed to have a significantly shorter length of hospital stay compared to IMEP plus L-ASP. IMEP plus PEG-ASP also showed a trend towards better clinical outcomes compared to IMEP plus L-ASP in NTCL.

PEG-ASP was first approved in 1994 for use in ALL patients who had developed hypersensitivity to native E. coli L-ASP [27]. PEG-ASP is potentially less immunogenic than native E. coli L-ASP and has a longer half-life, which allows one injection of PEG-ASP to replace multiple injections of native E. coli L-ASP. However, PEG-ASP is also associated with significantly higher median nadir serum asparaginase activity levels compared to native E. coli L-ASP [16]. Since toxicities such as hyperglycemia and thrombosis are caused by asparagine depletion $[28,29]$, there have been concerns that asparaginase-related toxicity could occur more frequently with PEG-ASP, and that toxicities may last longer due to the longer half-life of this preparation. In our study, the frequency of asparaginase-related toxicity was not significantly different between the L-ASP and PEG-ASP groups, except for hypofibrinogenemia, which occurred more frequently in the L-ASP group. Our study results were similar to the results of a phase 3 trial that compared native E. coli L-ASP with PEG-ASP in childhood ALL as a part of the postinduction regimen [16].

Although the changes in levels of hepatic enzymes, bilirubin, and albumin before and after chemotherapy were similar between two groups, the pattern of asparaginase-related toxicity was significantly different between the L-ASP and PEG-ASP groups after the first cycle. Patients displayed significantly elevated levels of AST, ALT, ALP, and t-bil, and a decrease in albumin levels after the first cycle of IMEP plus PEG-ASP. If a patient experienced significant hepatotoxicity due to increased AST, ALT, ALP, and t-bil levels or the decrease in albumin and fibrinogen, the subsequent cycle of chemotherapy would be delayed until the patient recovered from the toxicities. This could be an important point to consider when choosing the preparation of asparaginase because sustained asparagine depletion is more affected by dose intervals than by the dose per se, due to the anti-leukemic 
effect, which is dependent on the duration of asparagine depletion [15]. However, the frequency of asparaginaserelated toxicities in a total of 131 cycles between L-ASP and PEG-ASP did not show a significant difference except hyperglycemia and allergy which occurred more frequently in L-ASP group.

In our study, some patients were able to receive IMEP plus PEG-ASP at an outpatient clinic which enabled PEG-ASP to significantly shorten the length of hospital stay, without increasing the number of visits to the ER, as compared to L-ASP. However, it was easier to adjust the schedule and dose of native E. coli L-ASP when toxicity occurred, as native E. coli L-ASP was given on multiple days, whereas PEG-ASP was only given on day 1 of each cycle. In our study, native E. coli L-ASP was given in combination with IMEP, according to the planned treatment course in only 62 cycles, whereas PEG-ASP was given in combination with IMEP according to the planned treatment course in all cycles. A tendency towards more favorable outcome was shown in IMEP plus PEG-ASP, compared to IMEP plus L-ASP, with regard to the $\mathrm{CR}$ rate.

The current study had several limitations. Firstly, it only included a small number of patients, which makes it difficult to apply the results of this study to a general NTCL population. Secondly, it was a retrospective study and the choice of asparaginase preparation was made by the primary physician. This could have created selection bias in the study results. However, when considering that the incidence of NTCL only accounts for $6.3 \%$ of all non-Hodgkin's lymphoma in Korea, it is not easy to conduct a prospective trial with an adequate number of patients. Moreover, since the two groups of NTCL patients had similar baseline clinical characteristics and received the same IMEP regimen, the difference in toxicity was mostly dependent on the asparaginase preparation. Despite these limitations, this is the first study to report that the toxicity patterns of IMEP plus L-ASP and IMEP plus PEG-ASP are different after the first cycle of chemotherapy. Moreover, because reports of asparaginase- related toxicities of L-ASP compared with PEG-ASP in NTCL populations are lacking, the results of the current study are highly valuable. Lastly, the pharmacoeconomic implications of the two drugs, such as length of hospital stay, is clearly shown in the current study.

In conclusion, the frequency of asparaginase-related toxicity did not differ between the L-ASP and PEG-ASP groups in NTCL patients, except for hypofibrinogenemia, which occurred more frequently in patients taking L-ASP. The level of change in AST, ALT, ALP, t-bil, and albumin after chemotherapy was also similar between the two groups. IMEP plus PEG-ASP is more convenient than IMEP plus L-ASP for patients, because it can be given at an outpatient clinic which seemed to significantly shorten the length of hospital stay without increasing the number of visits to the ER. IMEP plus PEG-ASP also showed a tendency towards favorable clinical outcomes in terms of CR rate. Therefore, PEG-ASP is effective to a similar extent, and no more toxic than native $E$. coli L-ASP when used as a part of chemotherapy regimen with IMEP in NTCL, and is also associated with shorter hospital stays. Based on the results of this current study, IMEP plus PEG-ASP might be feasible as a front-line treatment of NTCL.

\section{Electronic Supplementary Material}

Supplementary materials are available at Cancer Research and Treatment website (http://www.e-crt.org).

\section{Conflicts of Interest}

Conflict of interest relevant to this article was not reported.

\section{Acknowledgments}

This study was supported by a grant from the Innovative Research Institute for Cell Therapy, Republic of Korea (A062260).

\section{References}

1. Sabattini E, Bacci F, Sagramoso C, Pileri SA. WHO classification of tumours of haematopoietic and lymphoid tissues in 2008: an overview. Pathologica. 2010;102:83-7.

2. Kwong YL, Chan AC, Liang R, Chiang AK, Chim CS, Chan TK, et al. CD56+ NK lymphomas: clinicopathological features and prognosis. Br J Haematol. 1997;97:821-9.

3. Yamaguchi M, Kita K, Miwa H, Nishii K, Oka K, Ohno T, et al. Frequent expression of P-glycoprotein/MDR1 by nasal
T-cell lymphoma cells. Cancer. 1995;76:2351-6.

4. Kwong YL, Kim WS, Lim ST, Kim SJ, Tang T, Tse E, et al. SMILE for natural killer/T-cell lymphoma: analysis of safety and efficacy from the Asia Lymphoma Study Group. Blood. 2012;120:2973-80.

5. Yamaguchi M, Kwong YL, Kim WS, Maeda Y, Hashimoto C, Suh C, et al. Phase II study of SMILE chemotherapy for newly diagnosed stage IV, relapsed, or refractory extranodal natural 
killer (NK)/T-cell lymphoma, nasal type: the NK-Cell Tumor Study Group study. J Clin Oncol. 2011;29:4410-6.

6. Jaccard A, Gachard N, Marin B, Rogez S, Audrain M, Suarez F, et al. Efficacy of L-asparaginase with methotrexate and dexamethasone (AspaMetDex regimen) in patients with refractory or relapsing extranodal NK/ T-cell lymphoma, a phase 2 study. Blood. 2011;117:1834-9.

7. Tong WH, Pieters R, Kaspers GJ, te Loo DM, Bierings MB, van den Bos C, et al. A prospective study on drug monitoring of PEGasparaginase and Erwinia asparaginase and asparaginase antibodies in pediatric acute lymphoblastic leukemia. Blood. 2014;123:2026-33.

8. Pieters R, Hunger SP, Boos J, Rizzari C, Silverman L, Baruchel A, et al. L-asparaginase treatment in acute lymphoblastic leukemia: a focus on Erwinia asparaginase. Cancer. 2011;117: 238-49.

9. Silverman LB, Gelber RD, Dalton VK, Asselin BL, Barr RD, Clavell LA, et al. Improved outcome for children with acute lymphoblastic leukemia: results of Dana-Farber Consortium Protocol 91-01. Blood. 2001;97:1211-8.

10. Kearney SL, Dahlberg SE, Levy DE, Voss SD, Sallan SE, Silverman LB. Clinical course and outcome in children with acute lymphoblastic leukemia and asparaginase-associated pancreatitis. Pediatr Blood Cancer. 2009;53:162-7.

11. Grace RF, Dahlberg SE, Neuberg D, Sallan SE, Connors JM, Neufeld EJ, et al. The frequency and management of asparaginase-related thrombosis in paediatric and adult patients with acute lymphoblastic leukaemia treated on Dana-Farber Cancer Institute consortium protocols. Br J Haematol. 2011;152:452-9.

12. Henriksen LT, Harila-Saari A, Ruud E, Abrahamsson J, Pruunsild K, Vaitkeviciene G, et al. PEG-asparaginase allergy in children with acute lymphoblastic leukemia in the NOPHO ALL2008 protocol. Pediatr Blood Cancer. 2015;62:427-33.

13. Vrooman LM, Stevenson KE, Supko JG, O'Brien J, Dahlberg $\mathrm{SE}$, Asselin BL, et al. Postinduction dexamethasone and individualized dosing of Escherichia coli L-asparaginase each improve outcome of children and adolescents with newly diagnosed acute lymphoblastic leukemia: results from a randomized study: Dana-Farber Cancer Institute ALL Consortium Protocol 00-01. J Clin Oncol. 2013;31:1202-10.

14. Park YK, Abuchowski A, Davis S, Davis F. Pharmacology of Escherichia coli-L-asparaginase polyethylene glycol adduct. Anticancer Res. 1981;1:373-6.

15. Stock W, Douer D, DeAngelo DJ, Arellano M, Advani A, Damon L, et al. Prevention and management of asparaginase/pegasparaginase-associated toxicities in adults and older adolescents: recommendations of an expert panel. Leuk Lymphoma. 2011;52:2237-53.

16. Place AE, Stevenson KE, Vrooman LM, Harris MH, Hunt SK, $\mathrm{O}$ 'Brien JE, et al. Intravenous pegylated asparaginase versus intramuscular native Escherichia coli L-asparaginase in newly diagnosed childhood acute lymphoblastic leukaemia (DFCI 05-001): a randomised, open-label phase 3 trial. Lancet Oncol. 2015;16:1677-90.

17. Avramis VI, Sencer S, Periclou AP, Sather H, Bostrom BC,
Cohen LJ, et al. A randomized comparison of native Escherichia coli asparaginase and polyethylene glycol conjugated asparaginase for treatment of children with newly diagnosed standard-risk acute lymphoblastic leukemia: a Children's Cancer Group study. Blood. 2002;99:1986-94.

18. Bi XW, Xia Y, Zhang WW, Sun P, Liu PP, Wang Y, et al. Radiotherapy and PGEMOX/GELOX regimen improved prognosis in elderly patients with early-stage extranodal NK/T-cell lymphoma. Ann Hematol. 2015;94:1525-33.

19. Wang JH, Wang L, Liu CC, Xia ZJ, Huang HQ, Lin TY, et al. Efficacy of combined gemcitabine, oxaliplatin and pegaspargase (P-gemox regimen) in patients with newly diagnosed advanced-stage or relapsed / refractory extranodal NK/T-cell lymphoma. Oncotarget. 2016;7:29092-101.

20. Asselin BL, Whitin JC, Coppola DJ, Rupp IP, Sallan SE, Cohen HJ. Comparative pharmacokinetic studies of three asparaginase preparations. J Clin Oncol. 1993;11:1780-6.

21. Muller HJ, Loning L, Horn A, Schwabe D, Gunkel M, Schrappe M, et al. Pegylated asparaginase (Oncaspar) in children with ALL: drug monitoring in reinduction according to the ALL/NHL-BFM 95 protocols. Br J Haematol. 2000;110: 379-84.

22. Panetta JC, Gajjar A, Hijiya N, Hak LJ, Cheng C, Liu W, et al. Comparison of native E. coli and PEG asparaginase pharmacokinetics and pharmacodynamics in pediatric acute lymphoblastic leukemia. Clin Pharmacol Ther. 2009;86:651-8.

23. Pileri SA, Grogan TM, Harris NL, Banks P, Campo E, Chan JK, et al. Tumours of histiocytes and accessory dendritic cells: an immunohistochemical approach to classification from the International Lymphoma Study Group based on 61 cases. Histopathology. 2002;41:1-29.

24. Kim SJ, Yoon DH, Jaccard A, Chng WJ, Lim ST, Hong H, et al. A prognostic index for natural killer cell lymphoma after nonanthracycline-based treatment: a multicentre, retrospective analysis. Lancet Oncol. 2016;17:389-400.

25. Lee J, Suh C, Park YH, Ko YH, Bang SM, Lee JH, et al. Extranodal natural killer T-cell lymphoma, nasal-type: a prognostic model from a retrospective multicenter study. J Clin Oncol. 2006;24:612-8.

26. Cheson BD, Pfistner B, Juweid ME, Gascoyne RD, Specht L, Horning SJ, et al. Revised response criteria for malignant lymphoma. J Clin Oncol. 2007;25:579-86.

27. Dinndorf PA, Gootenberg J, Cohen MH, Keegan P, Pazdur R. FDA drug approval summary: pegaspargase (oncaspar) for the first-line treatment of children with acute lymphoblastic leukemia (ALL). Oncologist. 2007;12:991-8.

28. Howard SC, Pui CH. Endocrine complications in pediatric patients with acute lymphoblastic leukemia. Blood Rev. 2002;16:225-43.

29. Hongo T, Okada S, Ohzeki T, Ohta H, Nishimura S, Hamamoto K, et al. Low plasma levels of hemostatic proteins during the induction phase in children with acute lymphoblastic leukemia: a retrospective study by the JACLS. Japan Association of Childhood Leukemia Study. Pediatr Int. 2002; 44:293-9. 